\title{
On spectral properties of linear combinations of idempotents
}

\author{
by \\ Hong-Ke Du (Xi'an), Chun-Yan Deng (Xi'an), \\ Mostafa Mbekhta (Lille) and Vladimír Müller (Praha)
}

\begin{abstract}
Let $P, Q$ be two linear idempotents on a Banach space. We show that the closedness of the range and complementarity of the kernel (range) of linear combinations of $P$ and $Q$ are independent of the choice of coefficients. This generalizes known results and shows that many spectral properties of linear combinations do not depend on their coefficients.
\end{abstract}

The non-singularity of the difference and sum of two idempotent matrices $P$ and $Q$ was first studied in [KRS]. In [BB] it was proved that the non-singularity of $P+Q$ is equivalent to the non-singularity of any linear combination $c_{1} P+c_{2} Q$ where $c_{1}, c_{2} \neq 0, c_{1}+c_{2} \neq 0$. The result was further generalized [DYD] to Hilbert space operators, and in [KR1] the stability of the nullity and rank of linear combinations of idempotents was proved.

Finally, in [KR2] it was proved (for Banach space operators) that the Fredholmness and semi-Fredholmness of linear combinations of two idempotents is independent of the choice of their coefficients.

We improve these results and show that for two idempotents $P, Q$ on a Banach space the closedness of the range of $c_{1} P+c_{2} Q$ and the complementarity of its kernel and range are independent of the choice of the coefficients $c_{1}, c_{2}$. Moreover, the kernel and range are continuous in the gap topology. This implies the independence of many spectral properties of linear combinations $c_{1} P+c_{2} Q$ from the coefficients $c_{1}, c_{2}$.

Let $T \in B(X)$ where $B(X)$ denotes the set of all bounded linear operators on a Banach space $X$. Denote by $N(T)$ and $R(T)$ the kernel and range of $T$, respectively.

2000 Mathematics Subject Classification: Primary 47B99; Secondary 47A56, 15A99.

Key words and phrases: linear combinations of idempotents, closed range, complemented subspaces.

The last author was supported by grant No. 201/06/0128 of GA ČR and by the Institutional Research Plan AV 0Z 10190503. 
An operator $P \in B(X)$ is called an idempotent if $P^{2}=P$. Note that the range of an idempotent is always closed since $R(P)=N(I-P)$, where $I$ is the identity operator.

The main result of this paper is the following theorem:

Main Theorem. Let $P, Q \in B(X)$ be idempotents. Let $c_{1}, c_{2} \in \mathbb{C} \backslash\{0\}$, $c_{1}+c_{2} \neq 0$. If $c_{1} P+c_{2} Q$ is invertible (left invertible, right invertible, injective, bounded below, surjective, Fredholm, upper semi-Fredholm, lower semi-Fredholm, left essentially invertible, right essentially invertible or has a generalized inverse, respectively), then $z_{1} P+z_{2} Q$ has the same property for all $z_{1}, z_{2} \in \mathbb{C} \backslash\{0\}, z_{1}+z_{2} \neq 0$.

Let $M, L$ be closed subspaces of a Banach space $X$. Let

$$
\delta(M, L)=\sup \{\operatorname{dist}\{x, L\}: x \in M,\|x\| \leq 1\} .
$$

The gap $\widehat{\delta}(M, L)$ between $M$ and $L$ is defined by

$$
\widehat{\delta}(M, L)=\max \{\delta(M, L), \delta(L, M)\} .
$$

The reduced minimum modulus of an operator $T \in B(X)$ is defined by

$$
\gamma(T)=\inf \{\|T x\|: \operatorname{dist}\{x, N(T)\} \leq 1\} .
$$

The most important property of the reduced minimum modulus is that $\gamma(T)>0$ if and only if $T$ has closed range. For basic properties of the gap and reduced minimum modulus see [K, pp. 197-201], or [M, Sec. 10].

Let $P, Q \in B(X)$ be idempotents. It is easy to see that instead of the function $\left(c_{1}, c_{2}\right) \mapsto c_{1} P+c_{2} Q$ of two variables $\left(c_{1}, c_{2}\right), c_{1}, c_{2} \neq 0, c_{1}+c_{2} \neq 0$, it is sufficient to study the function $z \mapsto P-z Q$ where $z \neq 0,1$.

For $z, z^{\prime} \in \mathbb{C} \backslash\{0,1\}$ write

$$
V_{z, z^{\prime}}=I+\frac{z-z^{\prime}}{z\left(z^{\prime}-1\right)} P .
$$

Lemma 1. Let $z, z^{\prime} \in \mathbb{C} \backslash\{0,1\}$. Then:

(i) $V_{z, z^{\prime}} V_{z^{\prime}, z}=V_{z^{\prime}, z} V_{z, z^{\prime}}=I$;

(ii) $V_{z, z^{\prime}} N(P-z Q)=N\left(P-z^{\prime} Q\right)$;

(iii) $\delta\left(N(P-z Q), N\left(P-z^{\prime} Q\right) \leq\|P\| \cdot\left|\frac{z-z^{\prime}}{z\left(z^{\prime}-1\right)}\right|\right.$.

Proof. (i) Clearly $V_{z, z^{\prime}} V_{z^{\prime}, z}=V_{z^{\prime}, z} V_{z, z^{\prime}}$ and

$$
\begin{aligned}
V_{z, z^{\prime}} V_{z^{\prime}, z} & =\left(I+\frac{z-z^{\prime}}{z\left(z^{\prime}-1\right)} P\right)\left(I+\frac{z^{\prime}-z}{z^{\prime}(z-1)} P\right) \\
& =I+\left(\frac{z-z^{\prime}}{z\left(z^{\prime}-1\right)}+\frac{z^{\prime}-z}{z^{\prime}(z-1)}+\frac{\left(z-z^{\prime}\right)\left(z^{\prime}-z\right)}{z z^{\prime}(z-1)\left(z^{\prime}-1\right)}\right) P \\
& =I+\frac{\left(z-z^{\prime}\right)}{z z^{\prime}(z-1)\left(z^{\prime}-1\right)}\left(z^{\prime}(z-1)-z\left(z^{\prime}-1\right)+z^{\prime}-z\right) P=I .
\end{aligned}
$$


(ii) Let $x \in N(P-z Q),\|x\|=1$. Then $Q x=\frac{1}{z} P X$ and $Q P x=P x$. We have

$$
\begin{aligned}
\left(P-z^{\prime} Q\right) V_{z, z^{\prime}} x & =P x+\frac{z-z^{\prime}}{z\left(z^{\prime}-1\right)} P x-\frac{z^{\prime}}{z} P x-\frac{z^{\prime}\left(z-z^{\prime}\right)}{z\left(z^{\prime}-1\right)} P x \\
& =\left(\frac{z z^{\prime}-z+z-z^{\prime}-z^{\prime 2}+z^{\prime}-z^{\prime} z+z^{\prime 2}}{z\left(z^{\prime}-1\right)}\right) P x=0 .
\end{aligned}
$$

Hence

$$
V_{z, z^{\prime}} N(P-z Q) \subset N\left(P-z^{\prime} Q\right) .
$$

Similarly, $V_{z^{\prime}, z} N\left(P-z^{\prime} Q\right) \subset N(P-z Q)$ and

$$
N\left(P-z^{\prime} Q\right)=V_{z, z^{\prime}} V_{z^{\prime}, z} N\left(P-z^{\prime} Q\right) \subset V_{z, z^{\prime}} N(P-z Q) .
$$

Hence $V_{z, z^{\prime}} N(P-z Q)=N\left(P-z^{\prime} Q\right)$.

(iii) Let $x \in N(P-z Q),\|x\|=1$. By (ii), $V_{z, z^{\prime}} x \in N\left(P-z^{\prime} Q\right)$, and so $\operatorname{dist}\left\{x, N\left(P-z^{\prime} Q\right)\right\} \leq\left\|x-V_{z, z^{\prime}} x\right\| \leq\left\|\frac{z-z^{\prime}}{z\left(z^{\prime}-1\right)} P x\right\| \leq\|P\| \cdot\left|\frac{z-z^{\prime}}{z\left(z^{\prime}-1\right)}\right|$, proving (iii).

Corollary 2. The function $z \mapsto N(P-z Q)$ is continuous in the gap topology for $z \in \mathbb{C} \backslash\{0,1\}$. Consequently, $z \mapsto \operatorname{dim} N(P-z Q)$ is constant for $z \in \mathbb{C} \backslash\{0,1\}$.

Proposition 3. Let $P, Q \in B(X)$ be idempotents. Let $z \in \mathbb{C} \backslash\{0,1\}$ and $0<\varepsilon<1 / 3$. Then there exists a neighbourhood $U$ of $z$ such that

$$
\frac{1}{1+\varepsilon} \gamma(P-z Q) \leq \gamma\left(P-z^{\prime} Q\right) \leq(1+\varepsilon) \gamma(P-z Q)
$$

for all $z^{\prime} \in U$.

Proof. Let $U$ be the set of all $z^{\prime} \in \mathbb{C} \backslash\{0,1\}$ such that

$$
\widehat{\delta}\left(N(P-z Q), N\left(P-z^{\prime} Q\right)\right)<\varepsilon / 6
$$

and

$$
\begin{aligned}
\left|z-z^{\prime}\right|< & \frac{\varepsilon}{6 \max \{1,\|P\|,\|Q\|\}} \\
& \times \min \left\{\left|z\left(z^{\prime}-1\right)\right|,\left|z^{\prime}(z-1)\right|,\left|\frac{z\left(z^{\prime}-1\right)}{z^{\prime}}\right|,\left|\frac{z^{\prime}(z-1)}{z}\right|\right\} .
\end{aligned}
$$

It is sufficient to show that $\gamma\left(P-z^{\prime} Q\right) \leq(1+\varepsilon) \gamma(P-z Q)$ for all $z^{\prime} \in U$ since the conditions are symmetrical in $z$ and $z^{\prime}$. 
Let $z^{\prime} \in U$. Let $\left(x_{n}\right)$ be a sequence of vectors in $X$ satisfying

$$
\operatorname{dist}\left\{x_{n}, N(P-z Q)\right\}=1
$$

for all $n$ and $\left\|(P-z Q) x_{n}\right\| \rightarrow \gamma(P-z Q)$. Without loss of generality we may assume that $\left\|x_{n}\right\| \rightarrow 1$.

For each $n$ set $x_{n}^{\prime}=V_{z, z^{\prime}} x_{n}$. We have

$$
\begin{aligned}
\limsup _{n \rightarrow \infty} \| & \left(P-z^{\prime} Q\right) x_{n}^{\prime} \| \\
= & \limsup _{n \rightarrow \infty}\left\|P x_{n}-z^{\prime} Q x_{n}+\frac{z-z^{\prime}}{z\left(z^{\prime}-1\right)} P x_{n}-\frac{z^{\prime}\left(z-z^{\prime}\right)}{z\left(z^{\prime}-1\right)} Q P x_{n}\right\| \\
= & \limsup _{n \rightarrow \infty} \|\left(P x_{n}-z Q x_{n}\right)+\left(z-z^{\prime}\right) Q x_{n}+\frac{z-z^{\prime}}{z\left(z^{\prime}-1\right)} P x_{n} \\
& -\frac{z^{\prime}\left(z-z^{\prime}\right)}{z^{\prime}-1} Q x_{n}+\frac{z^{\prime}\left(z-z^{\prime}\right)}{z\left(z^{\prime}-1\right)}\left(z Q x_{n}-Q P x_{n}\right) \| \\
\leq & \gamma(P-z Q)+\|Q\|\left|\frac{z^{\prime}\left(z-z^{\prime}\right)}{z\left(z^{\prime}-1\right)}\right| \gamma(P-z Q) \\
& +\left|\frac{z-z^{\prime}}{z^{\prime}-1}\right| \limsup _{n \rightarrow \infty}\left\|\left(z^{\prime}-1\right) Q x_{n}+\frac{P x_{n}}{z}-z^{\prime} Q x_{n}\right\| \\
\leq & (1+\varepsilon / 6) \gamma(P-z Q)+\left|\frac{z-z^{\prime}}{z\left(z^{\prime}-1\right)}\right| \limsup _{n \rightarrow \infty}\left\|P x_{n}-z Q x_{n}\right\| \\
\leq & (1+\varepsilon / 3) \gamma(P-z Q) .
\end{aligned}
$$

We now estimate $\operatorname{dist}\left\{x_{n}^{\prime}, N\left(P-z^{\prime} Q\right)\right\}$. For all $n$ large enough we have

$$
\begin{aligned}
\operatorname{dist}\left\{x_{n}^{\prime}, N\left(P-z^{\prime} Q\right)\right\} & \geq \operatorname{dist}\left\{x_{n}, N\left(P-z^{\prime} Q\right)\right\}-\left\|x_{n}-x_{n}^{\prime}\right\| \\
& \geq \operatorname{dist}\left\{x_{n}, N\left(P-z^{\prime} Q\right)\right\}-\varepsilon / 6 .
\end{aligned}
$$

For each $n$ there is a $y_{n} \in N\left(P-z^{\prime} Q\right)$ with

$$
\left\|x_{n}-y_{n}\right\|<\operatorname{dist}\left\{x_{n}, N\left(P-z^{\prime} Q\right)\right\}+1 / n \leq\left\|x_{n}\right\|+1 / n .
$$

Hence

$$
\begin{aligned}
1= & \operatorname{dist}\left\{x_{n}, N(P-z Q)\right\} \leq\left\|x_{n}-y_{n}\right\|+\operatorname{dist}\left\{y_{n}, N(P-z Q)\right\} \\
\leq & \left\|x_{n}-y_{n}\right\|+\left\|y_{n}\right\| \delta\left(N\left(P-z^{\prime} Q\right), N(P-z Q)\right) \\
\leq & \operatorname{dist}\left\{x_{n}, N\left(P-z^{\prime} Q\right)\right\}+1 / n \\
& +\left(2\left\|x_{n}\right\|+1 / n\right) \delta\left(N\left(P-z^{\prime} Q\right), N(P-z Q)\right)
\end{aligned}
$$

and

$\liminf _{n \rightarrow \infty} \operatorname{dist}\left\{x_{n}, N\left(P-z^{\prime} Q\right)\right\} \geq 1-2 \delta\left(N\left(P-z^{\prime} Q\right), N(P-z Q)\right) \geq 1-\varepsilon / 3$. 
Hence

$$
\liminf _{n \rightarrow \infty} \operatorname{dist}\left\{x_{n}^{\prime}, N\left(P-z^{\prime} Q\right)\right\} \geq 1-\varepsilon / 2
$$

and

$$
\gamma\left(P-z^{\prime} Q\right) \leq \frac{1+\varepsilon / 3}{1-\varepsilon / 2} \gamma(P-z Q) \leq(1+\varepsilon) \gamma(P-z Q) .
$$

Corollary 4. The function $z \mapsto \gamma(P-z Q)$ is continuous in $\mathbb{C} \backslash\{0,1\}$. The set $\{z \in \mathbb{C} \backslash\{0,1\}: \gamma(P-z Q)=0\}$ is both open and closed, so it is either empty or equal to $\mathbb{C} \backslash\{0,1\}$.

Proof. Follows from the previous proposition and the connectivity of $\mathbb{C} \backslash\{0,1\}$.

Recall that a closed subspace $M$ of a Banach space $X$ is called complemented if there exists a closed subspace $L \subset X$ such that $X=M \oplus L$. Equivalently, $M$ is complemented if and only if there exists a bounded linear idempotent $P \in B(X)$ with $R(P)=M$.

Corollary 5. Let $P, Q \in B(X)$ be idempotents. Let $z_{0} \in \mathbb{C} \backslash\{0,1\}$. Then:

(i) $\operatorname{dim} N(P-z Q)=\operatorname{dim} N\left(P-z_{0} Q\right)$ for all $z \in \mathbb{C} \backslash\{0,1\}$;

(ii) if $N\left(P-z_{0} Q\right)$ is complemented, then so is $N(P-z Q)$ for all $z \in$ $\mathbb{C} \backslash\{0,1\}$

(iii) if $R\left(P-z_{0} Q\right)$ is closed then so is $R(P-z Q)$ for all $z \in \mathbb{C} \backslash\{0,1\}$. Moreover, the function $z \mapsto R(P-z Q)$ is continuous in the gap topology. In particular, $\operatorname{codim} R(P-z Q)=\operatorname{codim} R\left(P-z_{0} Q\right)$;

(iv) if $R\left(P-z_{0} Q\right)$ is complemented then so is $R(P-z Q)$ for all $z \in$ $\mathbb{C} \backslash\{0,1\}$.

Proof. (i) was proved in Corollary 2.

(ii) By Lemma 1(ii), we have $N(P-z Q)=V_{z_{0}, z} N\left(P-z_{0} Q\right)$ where $V_{z_{0}, z}$ is an invertible operator. So $N(P-z Q)$ is complemented.

(iii) As $R\left(P-z_{0} Q\right)$ is closed, we have $\gamma\left(P-z_{0} Q\right)>0$ and, by Corollary 4 , $\gamma(P-z Q)>0$ for all $z \in \mathbb{C} \backslash\{0,1\}$. Hence $R(P-z Q)$ is closed. By Corollary 2 for $P^{*}, Q^{*} \in B\left(X^{*}\right)$, we have

$$
\begin{aligned}
\operatorname{codim} R(P-z Q) & =\operatorname{dim} N\left(P^{*}-z Q^{*}\right)=\operatorname{dim} N\left(P^{*}-z_{0} Q^{*}\right) \\
& =\operatorname{codim} R\left(P-z_{0} Q\right) .
\end{aligned}
$$

Similarly, the function $z \mapsto R(P-z Q)$ is continuous in the gap topology by duality.

(iv) Let $X=R\left(P-z_{0} Q\right) \oplus L_{0}$. Then $N\left(P^{*}-z_{0} Q^{*}\right)=R\left(P-z_{0} Q\right)^{\perp}$ and $X^{*}=N\left(P^{*}-z_{0} Q^{*}\right) \oplus L_{0}^{\perp}$. Note that $L_{0}^{\perp}$ is $w^{*}$-closed. By (ii), $N\left(P^{*}-z Q^{*}\right)$ 
is complemented in $X^{*}$. Moreover, by the proof of (ii), $N\left(P^{*}-z Q^{*}\right)=$ $V^{\prime} N\left(P^{*}-z_{0} Q^{*}\right)$ where $V^{\prime}=I+\frac{z_{0}-z}{z_{0}(z-1)} P^{*}$ is invertible. Hence $X^{*}=$ $N\left(P^{*}-z Q^{*}\right) \oplus L^{\prime}$ where $L^{\prime}=V^{\prime} L_{0}^{\perp}$ and $L^{\prime}$ is $w^{*}$-closed.

Let $L={ }^{\perp} L^{\prime}$. Since $R(P-z Q)^{\perp}+L^{\perp}=N\left(P^{*}-z Q^{*}\right)+L^{\prime}=X^{*}$, which is closed, $R(P-z Q)+L$ is a closed subspace of $X$ (see [LN, Theorem A.1.9]). We have

$$
(L \cap R(P-z Q))^{\perp}=L^{\perp}+R(P-z Q)^{\perp}=L^{\prime}+N\left(P^{*}-z Q^{*}\right)=X^{*},
$$

and so $L \cap R(P-z Q)=\{0\}$. Furthermore,

$$
(L+R(P-z Q))^{\perp}=L^{\perp} \cap R(P-z Q)^{\perp}=L^{\prime} \cap N\left(P^{*}-z Q^{*}\right)=\{0\},
$$

and so $L+R(P-z Q)=X$.

Hence $R(P-z Q)$ is complemented.

Recall that $T \in B(X)$ is left (right) invertible if there exists $S \in B(X)$ such that $S T=I$ (TS =I, respectively). It is well known that $T$ is left (right) invertible if and only if $T$ is injective and $R(T)$ is complemented ( $T$ is surjective and $N(T)$ is complemented, respectively). $T$ has a generalized inverse if there exists $S \in B(X)$ such that $T S T=T$. Equivalently, $T$ has a generalized inverse if and only if $T$ has closed range and both $N(T)$ and $R(T)$ are complemented.

$T \in B(X)$ is called upper (lower) semi-Fredholm if $R(T)$ is closed and $\operatorname{dim} N(T)<\infty(\operatorname{codim} R(T)<\infty$, respectively). $T$ is left (right) essentially invertible if there are $S, K \in B(X), K$ compact and $S T=I+K(T S=I+K$, respectively). It is well known that $T$ is left (right) essentially invertible if and only if $T$ is upper (lower) semi-Fredholm and $R(T)$ is complemented $(N(T)$ is complemented, respectively).

The Main Theorem is now an easy consequence of Corollary 5.

\section{References}

[BB] J. K. Baksalary and O. M. Baksalary, Nonsingularity of linear combinations of idempotent matrices, Linear Algebra Appl. 388 (2004), 25-29.

[DYD] H. K. Du, X. Y. Yao and C. Y. Deng, Invertibility of linear combinations of two idempotents, Proc. Amer. Math. Soc. 134 (2006), 1451-1457.

[K] T. Kato, Perturbation Theory for Linear Operators, 2nd ed., Springer, Berlin, 1976.

[KR1] J. J. Koliha and V. Rakočevič, The nullity and rank of linear combinations of idempotent matrices, Linear Algebra Appl. 418 (2006), 11-14.

[KR2] - - - Stability theorems for linear combinations of idempotents, Integral Equations Operator Theory, to appear.

[KRS] J. J. Koliha, V. Rakočevič and I. Straškraba, The difference and sum of projectors, Linear Algebra Appl. 388 (2004), 279-288. 
[LN] K. B. Laursen and M. M. Neumann, An Introduction to Local Spectral Theory, London Math. Soc. Monogr. 20, Oxford Sci. Publ., Clarendon Press, Oxford, 2000.

[M] V. Müller, Spectral Theory of Linear Operators, Oper. Theory Adv. Appl. 139, Birkhäuser, Basel, 2003.

College of Mathematics

and Information Science

Shaanxi Normal University

Xi'an 710062, P.R. China

E-mail: hkdu@snnu.edu.cn

cy-deng@263.net

Mathematical Institute

Czech Academy of Sciences

Žitná 25

11567 Praha 1, Czech Republic

E-mail: muller@math.cas.cz
Université Lille 1, UFR Mathématique 59655 Villeneuve d'Ascq, France E-mail: mbekhta@math.univ-lille1.fr

Received September 30, 2004

Revised version May 10, 2007 